\section{Utilização de adoçantes no Brasil: uma abordagem a partir de um inquérito domiciliar}

\section{Use of artificial sweeteners in Brazil: a household survey approach}

Utilización de edulcorantes en Brasil: un abordaje a partir de una encuesta domiciliaria
COMUNICAÇÃO BREVE

BRIEF COMMUNICATION

Paulo Sérgio Dourado Arrais 1

Marisa Perdigão de Negreiros Vianna 1

Anamaria Vargas Zaccolo 2

Luzia Izabel Mesquita Moreira 1

Patrícia Maria Pontes Thé 1

Ana Rosa Pinto Quidute 1

Andréia Turmina Fontanella 2

Tatiane da Silva Dal Pizzol 2

Noemia Urruth Leão Tavares 3

Maria Auxiliadora Oliveira 4

Vera Lucia Luiza 4

Luiz Roberto Ramos 5

Mareni Rocha Farias 6

Andréa Dâmaso Bertoldi 7

Sotero Serrate Mengue 2

doi: 10.1590/0102-311X00010719

\section{Correspondência}

P. S. D. Arrais

Universidade Federal do Ceará.

O objetivo foi estimar a prevalência do uso de adoçantes pela população adulta brasileira e características dos usuários. Análise de dados da Pesquisa Nacional de Acesso, Utilização e Promoção do Uso Racional de Medicamentos (PNAUM, 2014), um inquérito nacional de base populacional. $O$ desfecho de interesse foi o uso autorreferido de adoçantes entre brasileiros com 20 anos ou mais. As variáveis analisadas foram sexo, idade em anos completos, região do Brasil, escolaridade em anos completos e classificação econômica segundo o Critério Classificação Econômica Brasil da Associação Brasileira de Empresas de Pesquisa (ABEP). Os indicadores das condições de saúde foram: relato de doença crônica não transmissíveis (DCNT), número de DCNT e indice de massa corporal (IMC). A prevalência do uso de adoçantes na população adulta brasileira foi de 13,4\% (IC95\%: 12,5-14,3), sendo maior entre as pessoas do sexo feminino e no grupo com 60 anos ou mais, nas regiões Nordeste e Sudeste, entre pessoas da classe econômica $A / B$ e entre indivíduos obesos. As pessoas com doenças crônicas (em especial diabetes) foram as que mostraram maior prevalência de uso de adoçantes, sendo o uso maior quanto maior o número de comorbidades relatadas. A prevalência de uso de adoçantes foi de 13,4\% e mostrou-se associada a características sociodemográficas e de saúde.

Edulcorantes; Fatores Socioeconômicos; Inquéritos Epidemiológicos
Rua Pastor Samuel Monguba 1210, Fortaleza, CE 60430-327, Brasil.

parrais@ufc.br

1 Universidade Federal do Ceará, Fortaleza, Brasil

2 Universidade Federal do Rio Grande do Sul, Porto Alegre, Brasil.

3 Universidade de Brasília, Brasília, Brasil.

${ }_{4}^{4}$ Escola Nacional de Saúde Pública Sergio Arouca, Fundação Oswaldo Cruz, Rio de Janeiro, Brasil.

5 Universidade Federal de São Paulo, São Paulo, Brasil.

6 Universidade Federal de Santa Catarina, Florianópolis, Brasil.

7 Universidade Federal de Pelotas, Pelotas, Brasil. 


\section{Introdução}

A preferência pelo sabor doce inicia-se com o nascimento, persiste por toda a vida e pode ter relação com a obesidade, diabetes mellitus tipo II, resistência a insulina, síndrome metabólica, hipertensão arterial, doenças coronarianas, entre outras associadas ao consumo excessivo dos carboidratos 1 .

Os adoçantes dietéticos, ou adoçantes de mesa, como definido na regulamentação brasileira 2, são alternativas ao uso do açúcar refinado ${ }^{3}$. Eles conferem sabor doce aos alimentos e possuem baixa ou nenhuma caloria. Os adoçantes são usados principalmente por portadores de diabetes mellitus e obesidade, visando ao controle da ingesta calórica 3,4 .

Embora usados para o controle glicêmico e auxílio na prevenção e tratamento de morbidades, estudos abordam efeitos nocivos do seu consumo em longo prazo, tais como aumento de peso corporal, risco cardiometabólico, carcinogenicidade e potencial desenvolvimento de resistência a glicose 5 .

Considerando a ausência de estudos de abrangência nacional, foram utilizados dados da Pesquisa Nacional sobre Acesso, Utilização e Promoção do Uso Racional de Medicamentos (PNAUM), desenvolvida pelo Ministério da Saúde, para estimar a prevalência do uso de adoçantes pela população adulta brasileira e analisar sua distribuição segundo características dos usuários.

\section{Métodos}

Utilizaram-se dados da PNAUM, coletados de setembro/2013 a fevereiro/2014. A PNAUM utilizou amostra probabilística, segundo faixa etária e sexo, de residentes em zonas urbanas das cinco regiões do país, incluindo as capitais, com amostragem por conglomerados em três estágios (municípios, setores censitários e domicílios). Foram entrevistadas 41.433 pessoas que representam aproximadamente 171 milhões de brasileiros. Mais informações estão disponíveis em Mengue et al. 6.

Neste artigo, são analisados os dados das pessoas com idade $\geq 20$ anos $(\mathrm{N}=32.348)$. A prevalência de uso de adoçantes foi calculada considerando as pessoas que responderam "sim" à seguinte pergunta: “O(a) Sr(a) faz uso de algum adoçante na sua dieta?”.

As variáveis e respectivas categorizações analisadas são apresentadas na Tabela 1. O estado nutricional foi estimado pelo índice de massa corporal (IMC), calculado com base nos dados de peso e altura referidos pelos usuários 7 .

A análise descritiva apresenta as frequências relativas e os respectivos intervalos de $95 \%$ de confiança (IC95\%). Aplicou-se teste do qui-quadrado de Pearson para avaliação da significância estatística das diferenças entre os grupos, considerando o nível de significância de 5\%. As análises foram executadas com uso do pacote estatístico SPSS 18.0 (https://www.ibm.com/), empregando o conjunto de comandos CSPLAN apropriado para a análise de amostras complexas e garantindo a ponderação, de acordo com o desenho amostral.

O estudo foi aprovado pela Comissão Nacional de Ética em Pesquisa (parecer no 398.131, de 16 de setembro de 2013).

\section{Resultados}

A prevalência do uso de adoçantes dietéticos pela população brasileira acima dos vinte anos e residente em zona urbana foi de 13,4\% (IC95\%: 12,5-14,3), sendo maior entre as pessoas do sexo feminino, no grupo com 60 anos ou mais, entre os residentes das regiões Nordeste e Sudeste, pertencentes à classe econômica $\mathrm{A} / \mathrm{B}$, com excesso de peso e entre aquelas que referiram uma ou mais doenças crônicas (Tabela 1).

O uso de adoçantes foi maior entre os indivíduos que referiram diabetes como única doença ou associada a outras comorbidades (Tabela 2). 


\section{Tabela 1}

Prevalência do uso de adoçantes segundo variáveis demográficas, socioeconômicas, de assistência médica e condições de saúde. Pesquisa Nacional de Acesso, Utilização e Promoção do Uso Racional de Medicamentos, Brasil, 2014 (N = 32.348).

\begin{tabular}{|c|c|c|c|c|}
\hline Características da amostra & $\begin{array}{c}\% \\
\text { amostra }\end{array}$ & $\begin{array}{l}\text { Prevalência do uso de } \\
\text { adoçantes * }\end{array}$ & IC95\% & Valor de $p$ \\
\hline Sexo & & & & $<0,001$ \\
\hline Masculino & 46,3 & 9,3 & $8,3-10,3$ & \\
\hline Feminino & 53,7 & 16,9 & $15,9-18,0$ & \\
\hline Faixa etária (anos) & & & & $<0,001$ \\
\hline 20-39 & 45,3 & 10,3 & $8,8-11,9$ & \\
\hline $40-59$ & 36,0 & 20,3 & $18,6-22,1$ & \\
\hline 60 & 18,7 & 29,5 & $27,5-31,5$ & \\
\hline Região & & & & 0,02 \\
\hline Norte & 6,7 & 10,7 & $9,4-22,1$ & \\
\hline Nordeste & 23,4 & 14,7 & $13,3-16,3$ & \\
\hline Sudeste & 47,5 & 14,1 & $12,5-15,9$ & \\
\hline Sul & 14,7 & 11,3 & $9,9-12,9$ & \\
\hline Centro-oeste & 7,8 & 11,3 & $9,9-12,8$ & \\
\hline Escolaridade (anos) & & & & 0,144 \\
\hline $0-8$ & 58,3 & 12,8 & $11,8-14,0$ & \\
\hline $9-11$ & 30,6 & 14,2 & $12,9-15,5$ & \\
\hline 12 & 11,1 & 13,7 & $12,0-15,7$ & \\
\hline Classificação econômica ** & & & & $<0,001$ \\
\hline$A / B$ & 24,2 & 17,4 & $15,7-19,3$ & \\
\hline C & 55,1 & 13,3 & $12,2-14,5$ & \\
\hline $\mathrm{D} / \mathrm{E}$ & 20,7 & 8,9 & $7,9-10,1$ & \\
\hline IMC & & & & $<0,001$ \\
\hline Baixo & 2,6 & 6,2 & $4,0-9,4$ & \\
\hline Normal & 44,3 & 9,9 & $8,9-11,1$ & \\
\hline Sobrepeso & 37,5 & 15,0 & $13,9-16,2$ & \\
\hline Obeso & 15,6 & 22,7 & $20,4-25,2$ & \\
\hline Tem doença crônica & & & & $<0,001$ \\
\hline Sim & 39,7 & 24,2 & $22,8-25,8$ & \\
\hline Não & 60,3 & 6,4 & $5,7-7,2$ & \\
\hline Número de doenças crônicas & & & & $<0,001$ \\
\hline 0 & 60,3 & 6,7 & $5,9-7,5$ & \\
\hline 1 & 20,5 & 16,3 & $14,7-18,0$ & \\
\hline 2 & 9,9 & 28,7 & $26,3-31,3$ & \\
\hline $3+$ & 9,3 & 37,4 & $35,0-40,0$ & \\
\hline
\end{tabular}

IC95\%: intervalo de 95\% de confiança IMC: índice de massa corporal.

* Percentuais ponderados pelos pesos amostrais.

** A classificação econômica foi realizada segundo o Critério Classificação Econômica Brasil da Associação Brasileira das Empresas de Pesquisa (CCEB 2013/ABEP - http://www.abep.org/). 


\section{Tabela 2}

Prevalência do uso de adoçantes segundo as doenças crônicas declaradas e estado nutricional. Pesquisa Nacional de Acesso, Utilização e Promoção do Uso Racional de Medicamentos, Brasil, 2014 ( $\mathrm{N}=32.348)$.

\begin{tabular}{|c|c|c|c|}
\hline Doenças crônicas & $\begin{array}{l}\text { Prevalência da } \\
\text { condição }\end{array}$ & $\begin{array}{l}\text { Prevalência do uso de } \\
\text { adoçantes * }\end{array}$ & IC95\% \\
\hline Hipertensão & 23,7 & 27,3 & $25,6-29,2$ \\
\hline Diabetes & 6,8 & 60,5 & $57,5-63,5$ \\
\hline Hipercolesterolemia & 10,1 & 33,2 & $30,7-35,9$ \\
\hline Excesso de peso ** & 51,2 & 17,0 & $15,8-18,3$ \\
\hline Hipertensão + diabetes & 0,8 & 60,5 & $57,1-63,9$ \\
\hline Hipertensão + excesso de peso & 9,3 & 30,0 & $27,9-32,1$ \\
\hline Hipertensão + hipercolesterolemia & 1,2 & 37,2 & $34,3-40,1$ \\
\hline Hipertensão + diabetes + excesso de peso & 2,1 & 60,3 & $56,3-64,2$ \\
\hline Diabetes + excesso de peso & 0,9 & 59,2 & $55,8-62,6$ \\
\hline Diabetes + hipercolesterolemia & 0,2 & 60,1 & $55,3-64,8$ \\
\hline Hipercolesterolemia + excesso de peso & 2,0 & 35,6 & $32,6-38,6$ \\
\hline Hipertensão + diabetes + hipercolesterolemia + excesso de peso & 1,5 & 59,8 & $53,5-65,8$ \\
\hline
\end{tabular}

IC95\%: intervalo de 95\% de confiança.

* Percentuais ponderados pelos pesos amostrais;

** Sobrepeso + obesidade.

\section{Discussão}

O estudo possibilitou a identificação da prevalência do consumo de adoçantes no Brasil e suas características. Foi possível observar que o consumo é menor que o identificado em Pelotas, Rio Grande do Sul, Brasil (19\%) 3 e muito próximo ao encontrado nos Estados Unidos, 14,1\% (EP $\pm 0,8)$ 8. De maneira geral, e apesar das diferenças metodológicas, os resultados não diferem muito do que foi encontrado em outros estudos, em que dentre os maiores consumidores de adoçantes estão as mulheres 3,4,8,9,10,11, os idosos 3,4,8 e os demais indivíduos com maior nível de escolaridade 3,8,11, com maior poder econômico 3,8,10,11, com excesso de peso 3,8 e portadores de diabetes e hipertensão 3,8,11.

No que diz respeito à maior prevalência de consumo entre as mulheres, esse resultado pode estar relacionado a uma maior preocupação com o controle de peso e com o cuidado da saúde, além da preocupação estética socialmente construída. Entre os idosos, o envelhecimento, o ganho de peso associado e as doenças crônicas podem ser estímulos para o consumo de adoçantes 4 e orientações para hábitos saudáveis por parte dos profissionais de saúde 12 .

Com relação às maiores prevalências de uso encontradas nas regiões Nordeste e Sudeste, dados do Vigitel, 2017 (Vigilância de Fatores de Risco e Proteção para Doenças Crônicas por Inquérito Telefônico) revelaram que as maiores prevalências de hipertensão arterial e diabetes mellitus foram registradas nas capitais dos estados dessas regiões 13 . Apesar de esses resultados não serem representativos das regiões como um todo, demonstram a possível influência que essas doenças crônicas possam exercer nesse consumo.

Os resultados encontrados no presente estudo também mostram que o uso de adoçantes foi maior entre os indivíduos que apresentaram diabetes mellitus isolado ou associado a hipertensão, a hipercolesterolemia e a excesso de peso. Castro \& Franco 14 relataram que a prevalência no uso de adoçantes após o diagnóstico de diabetes mellitus passou de $6 \%$ a $90 \%$.

A maior prevalência de consumo de adoçante entre aqueles com excesso de peso em relação aos eutróficos, era esperado. O estímulo à adoção de estilos de vida saudáveis, incluindo o controle de peso pode ser uma das razões para o consumo 15,16 .

É importante observar que a substituição do açúcar pelo adoçante pode gerar a falsa ideia de total benefício e negligência de outras restrições e adaptações na dieta, acarretando ganho de peso com seu 
uso prolongado e isolado ${ }^{17}$. O consumo de adoçantes artificiais não calóricos pode, ainda, suscitar intolerância a glicose 18 .

Diversos estudos discutiram os benefícios e os riscos no uso de adoçantes em diferentes populações. Mais recentemente, revisões sistemáticas procuraram elucidar as divergências, no entanto, ainda há fragilidades nas evidências entre o consumo intenso de adoçantes e certos riscos 5,19.

Algumas limitações devem ser observadas. Não foram coletadas informações sobre a composição do adoçante utilizado, bem como sobre tempo e quantidade de uso. A pergunta sobre o uso de adoçantes não diferenciou os artificiais de naturais, como a sacarose. Também não considerou o consumo de alimentos que contenham adoçantes, como refrigerantes, sucos e sobremesas, não permitindo uma análise mais abrangente desse consumo. As informações de peso e altura, utilizadas para o cálculo do IMC, foram autorreferidas.

Este estudo preenche uma lacuna acerca do conhecimento dos hábitos de consumo dos brasileiros, que foi de aproximadamente $13 \%$, chegando a $60 \%$ entre a população diabética e mostrando-se associado ao IMC, com predomínio entre os obesos. Apesar de muitas pessoas considerarem esse uso como uma prática saudável, é preciso estar atento aos seus possíveis riscos. Desta forma, faz-se necessário estimular as pessoas a mudanças no estilo de vida, com adoção de planos alimentares adequados na qualidade e quantidade calórica, prática de atividade física e promoção na redução do estresse diário, que são pilares insubstituíveis para a promoção de saúde.

\section{Colaboradores}

P. S. D. Arrais e S. S. Mengue contribuíram na concepção, análise e interpretação dos resultados, redação do artigo e revisão crítica do conteúdo intelectual; e aprovação final da versão a ser publicada. M. P. N. Vianna, L. I. M. Moreira, P. M. P. Thé, A. R. P. Quidute, A. T. Fontanella, N. U. L. Tavares, M. A. Oliveira, V. L. Luiza, L. R. Ramos, M. R. Farias e A. D. Bertoldi contribuíram na análise e interpretação dos resultados, redação do artigo e revisão crítica do conteúdo intelectual. A. V. Zaccolo contribuiu na análise e interpretação dos resultados e redação do artigo. T. S. Dal Pizzol contribuiu na análise e interpretação dos resultados e revisão crítica do conteúdo intelectual.

\section{Informações adicionais}

ORCID: Paulo Sérgio Dourado Arrais (0000-00024502-8467); Marisa Perdigão de Negreiros Vianna (0000-0002-1773-8111); Anamaria Vargas Zaccolo (0000-0001-6195-9301); Luzia Izabel Mesquita Moreira (0000-0003-0873-639X); Patrícia Maria Pontes Thé (0000-0003-2298-0700); Ana Rosa Pinto Quidute (0000-0003-1876-6410); Andréia Turmina Fontanella (0000-0003-0455-9429); Tatiane da Silva Dal Pizzol (0000-0002-7566-7745); Noemia Urruth Leão Tavares (0000-0001-6180-7527); Maria Auxiliadora Oliveira (0000-0003-2400536X); Vera Lucia Luiza (0000-0001-6245-7522); Luiz Roberto Ramos (0000-0003-3143-8315); Mareni Rocha Farias (0000-0001-7166-8444); Andréa Dâmaso Bertoldi (0000-0002-4680-3197); Sotero Serrate Mengue (0000-0002-3349-8541).

\section{Agradecimentos}

Aos Departamentos de Ciência e Tecnologia (Decit) e de Assistência Farmacêutica (DAF) da Secretaria de Ciência, Tecnologia e Insumos Estratégicos (SCTIE) do Ministério da Saúde, pelo financiamento e apoio técnico para a realização da Pesquisa Nacional sobre Acesso, Utilização e Promoção do Uso Racional de Medicamentos. 


\section{Referências}

1. Schiffman SS, Graham BG, Sattely-Miller EA, Peterson-Dancy M. Elevated and sustained desire for sweet taste in African-Americans: a potential factor in the development of obesity. Nutrition 2000; 16:886-93.

2. Agência Nacional de Vigilância Sanitária. Resolução RDC no 271, de 22 de setembro de 2005. Aprova o "Regulamento Técnico Para Açúcares E Produtos Para Adoçar”. Diário Oficial da União 2005; 23 set.

3. Zanini RV, Araújo CL, Martínez-Mesa J. Utilização de adoçantes dietéticos entre adultos em Pelotas, Rio Grande do Sul, Brasil: um estudo de base populacional. Cad Saúde Pública 2011; 27:924-34.

4. Oliveira PB, Franco LJ. Consumo de adoçantes e produtos dietéticos por indivíduos com diabetes melito tipo 2, atendidos pelo Sistema Único de Saúde em Ribeirão Preto, SP. Arq Bras Endocrinol Metab 2010; 54:455-62.

5. Bruyère $\mathrm{O}$, Ahmed $\mathrm{SH}$, Atlan $\mathrm{C}$, Belegaud $\mathrm{J}$, Bortolotti M, Canivenc-Lavier M-C, et al. Review of the nutritional benefits and risks related to intense sweeteners. Arch Public Health 2015; 73:41.

6. Mengue SS, Bertoldi AD, Boing AC, Tavares NUL, Pizzol TSD, Oliveira MA, et al. National Survey on Access, Use and Promotion of Rational Use of Medicines (PNAUM): household survey component methods. Rev Saúde Pública 2016; 50 Suppl 2:4s.

7. World Health Organization. Physical status: the use and interpretation of anthropometry. Report of a WHO Expert Committee. Geneva: World Health Organization; 1995. (Technical Report Series, 854).

8. Sylvetsky AC, Jin Y, Clark EJ, Welsh JA, Rother KI, Talegawkar SA. Consumption of lowcalorie sweeteners among children and adults in the United States. J Acad Nutr Diet 2017; 117:441-448.e2.

9. Drewnowski A, Rehm CD. The use of low-calorie sweeteners is associated with self-reported prior intent to lose weight in a representative sample of US adults. Nutr Diabetes 2016; 6:e202.

10. Nielsen SJ, Popkin BM. Changes in beverage intake between 1977 and 2001. Am J Prev Med 2004; 27:205-10.
11. Geraldo APG, Pinto-e-Silva MEM. Nonnutritive sweeteners in Brazil: current use and associated factors. J Hum Growth Dev 2016; 26:297-306.

12. Flores TR, Nunes BP, Assunção MCF, Bertoldi AD. Hábitos saudáveis: que tipo de orientação a população idosa está recebendo dos profissionais de saúde? Rev Bras Epidemiol 2016; 19:167-80

13. Departamento de Vigilância de Doenças e Agravos não Transmissíveis e Promoção da Saúde, Secretaria de Vigilância em Saúde, Ministério da Saúde. Vigitel Brasil 2017: vigilância de fatores de risco e proteção para doenças crônicas por inquérito telefônico: estimativas sobre frequência e distribuição sociodemográfica de fatores de risco e proteção para doenças crônicas nas capitais dos 26 estados brasileiros e no Distrito Federal em 2017. Brasília: Ministério da Saúde; 2017.

14. Castro AGP, Franco LJ. Caracterização do consumo de adoçantes alternativos e produtos dietéticos por indivíduos diabéticos. Arq Bras Endocrinol Amp Metabol 2002; 46:280-7.

15. Flores TR, Gomes AP, Soares ALG, Nunes BP, Assunção MCF, Gonçalves H, et al. Aconselhamento por profissionais de saúde e comportamentos saudáveis entre idosos: estudo de base populacional em Pelotas, sul do Brasil, 2014. Epidemiol Serv Saúde 2018; 27:e201720112.

16. Filgueiras AR, Sawaya AL. Intervenção multidisciplinar e motivacional para tratamento de adolescentes obesos brasileiros de baixa renda: estudo piloto. Rev Paul Pediatr 2018; 36:18691.

17. Yang Q. Gain weight by "going diet?” Artificial sweeteners and the neurobiology of sugar cravings. Yale J Biol Med 2010; 83:101-8.

18. Suez J, Korem T, Zeevi D, Zilberman-Schapira G, Thaiss CA, Maza O, et al. Artificial sweeteners induce glucose intolerance by altering the gut microbiota. Nature 2014; 514:181-6.

19. Makarem N, Bandera EV, Nicholson JM, Parekh N. Consumption of sugars, sugary foods, and sugary beverages in relation to cancer risk: a systematic review of longitudinal studies. Annu Rev Nutr 2018; 38:17-39. 


\section{Abstract}

The objective was to estimate the prevalence of artificial sweetener use by the adult Brazilian population and users' characteristics. Analysis of data from the Brazilian National Survey on Access, Utilization, and Promotion of Rational Use of Medicines (PNAUM, 2014), a nationwide population-based survey. The target outcome was selfreported use of sweeteners by Brazilians 20 years and older. The independent variables were sex, age, major geographic region of Brazil, schooling in complete years, and economic status according to the Brazilian Economic Classification Criterion of the Brazilian Association of Research Companies $(A B E P)$. The health condition indicators were: self-reported noncommunicable diseases (NCDs), number of NCDs, and body mass index (BMI). Prevalence of sweetener use in the Brazilian adult population was $13.4 \%$ (95\%CI: 12.5-14.3), and it was higher in females and in persons 60 years or older, in the Northeast and Southeast, among individuals from economic classes $A$ and $B$, and among obese individuals. Persons with chronic diseases (especially diabetes) showed the highest prevalence of use of sweeteners, and their use increased with the number of reported comorbidities. Prevalence of use of artificial sweeteners was $13.4 \%$ and was associated with sociodemographic and health characteristics.

Sweetening; Socioeconomic Factors; Health Surveys

\section{Resumen}

El objetivo fue estimar la prevalencia del uso de edulcorantes por parte de la población adulta brasileña y las características de los usuarios. Análisis de datos de la Encuesta Nacional de Acceso, Utilización y Promoción del Uso Racional de Medicamentos (PNAUM, 2014), una encuesta nacional de base poblacional. El resultado de interés fue el uso autoinformado de edulcorantes entre brasileños con 20 años o más. Las variables analizadas fueron: sexo, edad (años completados), región de Brasil, escolaridad (años completados), así como la clasificación económica según el Criterio Clasificación Económica Brasil de la Asociación Brasileña de Empresas de Investigación (ABEP). Los indicadores de las condiciones de salud fueron: informe de enfermedades crónicas (DCNT), número de DCNT e índice de masa corporal (IMC). La prevalencia del uso de edulcorantes en la población adulta brasileña fue de un 13,4\% (IC95\%: 12,5-14,3), siendo mayor entre las personas de sexo femenino y en el grupo con 60 años o más, en las regiones Nordeste y Sudeste, entre personas de clase económica $A / B$ y entre individuos obesos. Las personas con enfermedades crónicas (en especial diabetes) fueron las que mostraron una mayor prevalencia de uso de edulcorantes, siendo el uso mayor, cuanto mayor fuera el número de comorbilidades informadas. Conclusiones: la prevalencia de uso de edulcorantes fue de un 13,4\% y se mostró asociada a características sociodemográficas y de salud.

Edulcorantes; Factores Socioeconómicos;

Encuestas Epidemiológicas
Recebido em 18/Jan/2019

Versão final reapresentada em 11/Ago/2019

Aprovado em 03/Set/2019 